\title{
Optimization Issues for a Micropulsed Plasma Thruster
}

\author{
Michael Keidar* and Iain D. Boyd ${ }^{\dagger}$ \\ University of Michigan, Ann Arbor, Michigan 48109 \\ Erik L. Antonsen ${ }^{\ddagger}$ and Rodney L. Burton ${ }^{\S}$ \\ University of Illinois at Urbana-Champaign, Illinois 61801 \\ and \\ Gregory G. Spanjers ${ }^{\text {IT }}$ \\ Air Force Research Laboratory, Kirtland Air Force Base, New Mexico 87117
}

\begin{abstract}
Several issues related to the design of a micropulsed plasma thruster ( $\mu$ PPT) are considered. It is concluded that the choice of the optimal energy level for a given $\mu$ PPT geometry is very important. If the discharge energy is small, propellant charring would limit the operational time of the thruster. It is found that the charring phenomenon is associated with nonuniformity (in the radial direction between the electrodes) in the propellant ablation rate. On the other hand, higher energy leads to discharge constriction on the positive electrode and causes azimuthal nonuniformity. Reasoning leading to such nonuniformity is considered, and general suggestions for optimal energy and thruster size selections are presented.
\end{abstract}

\section{Nomenclature}

$a=$ thermal diffusivity

$B \quad=$ self-magnetic field

$b \quad=$ specific inductance in the acceleration channel

$C_{p} \quad=$ specific heat

$e=$ electron charge

$g \quad=$ acceleration of gravity constant

$h=$ distance between the electrodes

$I=$ instantaneous total discharge current

$I_{\text {bit }} \quad=$ impulse bit

$I_{\text {out }}=$ current fraction outside the spot

$I_{s} \quad=$ current fraction in the spot

$I_{\mathrm{sp}} \quad=$ specific impulse

$j \quad=$ current density

$j_{a} \quad=$ anode current density

$j_{\text {eth }}=$ thermal electron current density

$j_{i} \quad=$ ion current density

$\ln \Lambda=$ Coulomb logarithm

$m \quad=$ heavy particle mass

$m_{e} \quad=$ electron mass

$n=$ plasma density

$n_{e a}=$ plasma density near the anode

$n_{\mathrm{er}} \quad=$ density of eroded particles

$n_{s} \quad=$ density at the surface

$n_{1}, n_{2}=$ densities

$P_{\mathrm{eq}}=$ equilibrium pressure

$Q_{F} \quad=$ heat due to particle convection

$Q_{j} \quad=$ Joule heat

$Q_{k} \quad=$ the kinetic energy

$Q_{r} \quad=$ radiation heat

Received 13 October 2004; revision received 13 April 2005; accepted for publication 30 May 2005. Copyright (C) 2005 by the American Institute of Aeronautics and Astronautics, Inc. All rights reserved. Copies of this paper may be made for personal or internal use, on condition that the copier pay the $\$ 10.00$ per-copy fee to the Copyright Clearance Center, Inc., 222 Rosewood Drive, Danvers, MA 01923; include the code 0748-4658/06 \$10.00 in correspondence with the CCC.

*Assistant Research Scientist, Department of Aerospace Engineering. Senior Member AIAA.

${ }^{\dagger}$ Professor, Department of Aerospace Engineering. Associate Fellow AIAA.

\#Postdoctoral Fellow, Department of Nuclear, Plasma, and Radiological Engineering. Member AIAA.

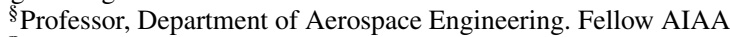

IProgram Manager, Space Vehicles Directorate. Member AIAA. $q_{a}=$ heat flux to the anode

$q(t)=$ heat flux

$T=$ propellant temperature

$T_{a} \quad=$ anode surface temperature

$T_{e} \quad=$ electron temperature

$T_{s} \quad=$ Teflon ${ }^{\circledR}$ surface temperature

$T_{\mathrm{sp}}=$ Teflon surface temperature at the end of the pulse

$T_{0} \quad=$ initial Teflon surface temperature, $300 \mathrm{~K}$

$T_{1}, T_{2}=$ temperatures

$t_{p} \quad=$ pulse duration

$U_{\text {sh }} \quad=$ sheath potential drop

$V \quad=$ plasma velocity

$V_{1} \quad=$ velocity at the Knudsen-layer edge

$Z_{i} \quad=$ ion mean charge

$\beta=$ Hall parameter

$\Gamma \quad=$ ablation rate, $\mathrm{kg} / \mathrm{m}^{2} \cdot \mathrm{s}$

$\Delta H=$ ablation heat

$\Delta M=$ ablation mass

$\lambda=$ thermal conductivity

$\mu \quad=$ permeability

$v_{\mathrm{ei}} \quad=$ electron-ion collision frequency

$\rho \quad=$ specific weight

$\sigma \quad=$ plasma conductivity

$\varphi \quad=$ electric potential

\section{Introduction}

$\mathbf{P}$ ULSED plasma thrusters (PPTs) are considered an attractive propulsion option for stationkeeping and drag makeup purposes for mass- and power-limited satellites that require micro-Newton second to milli-Newton second impulse bits. ${ }^{1,2}$ In particular, the U.S. Air Force has a growing interest in highly maneuverable microsatellites to perform various missions such as space-based surveillance, on-orbit servicing, inspection, and spacecraft control. ${ }^{1,2}$ PPTs have been developed since the early 1960s and were among the first electric propulsion concepts accepted for space flight. As a recent illustration of the maturity of this technology, an electromagnetic PPT was successfully operated for pitch axis control on the EO-1 spacecraft. ${ }^{3,4}$ It was shown that the PPT can be easily scaled down in power and size. A micro-PPT ( $\mu \mathrm{PPT})$ that is the miniature version of the traditional PPT has been designed for delivery of very small impulse bits. ${ }^{5,6}$ The $\mu$ PPT can deliver an impulse bit in the $10 \mu \mathrm{N} \cdot \mathrm{s}$ range to provide attitude control and stationkeeping for microsatellites. In this thruster, the discharge across the propellant surface ablates a portion of the propellant, ionizes it, and then accelerates it predominantly electromagnetically generating the thrust. 


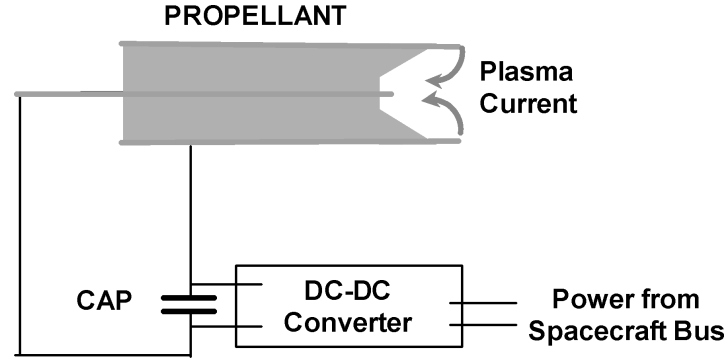

Fig. 1 Schematic of the $\mu$ PPT.

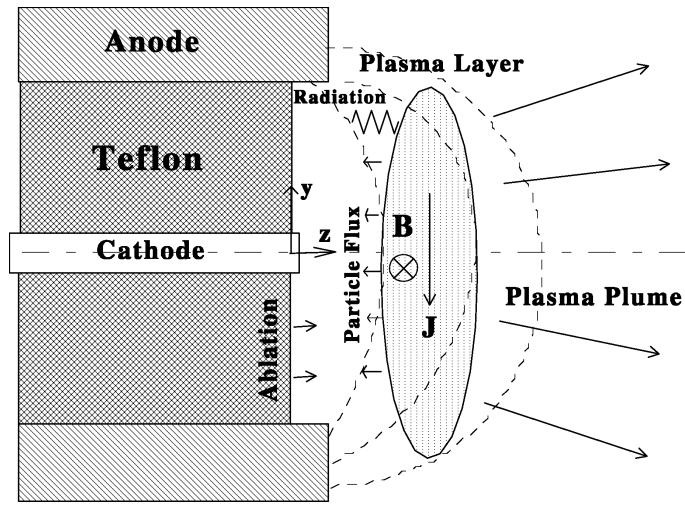

Fig. 2 Schematic of the coaxial $\mu$ PPT electrode configuration.

This thruster with power processing unit is shown schematically in Fig. 1. It is expected that the use of electromagnetic acceleration to create thrust will also lead to relatively high specific impulse. Even greater miniaturization of the PPT technology was shown recently by Simon and Land. ${ }^{7}$ They demonstrated $\mu$ PPT operation at the micromechanical systems scale with an interelectrode gap of only several hundred micrometers.

In this paper, we consider several issues related to miniaturization of a PPT. It will be shown that the choice of the optimal energy level for a given $\mu \mathrm{PPT}$ geometry is very important. If the discharge energy is small, propellant charring (carbonization) will limit the operational time of the thruster. The charring phenomenon is associated with nonuniformity (in the radial direction between the electrodes) in the propellant ablation rate. ${ }^{8}$ On the other hand, when the discharge energy is large, another discharge non-uniformity, or arc-spoking, occurs. ${ }^{9}$ The plasma typically covers the entire central electrode, but attaches at specific points on the outer electrode resulting in an azimuthal nonuniformity of the discharge. Therefore, the desirable thruster operational conditions (discharge energy) should be chosen by a tradeoff between these extreme cases with high and low discharge energy. It is, however, not clear what is the transition point to the extreme regime and what considerations should be involved in the mentioned trade study.

In this paper, a model for the tradeoff study is proposed. The rest of the paper is organized as follows. The models of the plasma layer and current constriction are described in Sec. II. Anode spot formation phenomenon is considered in Sec. III followed by a description of the thruster parameters and their changes and effects associated with discharge nonuniformity, such as ablated mass (Sec. IV), Teflon ${ }^{\circledR}$ temperature (Sec. V), propellant charring (Sec. VI), and thrust (Sec. VII).

\section{Modeling of Current Constriction in $\mu$ PPT}

\section{A. Plasma Layer Model}

A model of the plasma generation in a $\mu$ PPT has been described elsewhere ${ }^{8-10}$ In this paper, we briefly outline this model as shown schematically in Fig. 2. A fluid model is used because the plasma density near the propellant face is large, on the order of
$10^{23}-10^{24} \mathrm{~m}^{-3}$ (Refs. 8-10). Mechanisms of energy transfer from the plasma column to the wall of the Teflon include heat transfer by particle convection and by radiation. When it is taken into account that the gradients in the axial direction are relatively small, ${ }^{12,13}$ it is assumed that within the plasma layer all parameters vary only in the $y$ direction (Fig. 2). The electron energy balance equation can be written in the form

$$
\frac{3}{2}(n) \frac{\mathrm{d} T_{e}}{\mathrm{~d} t}=Q_{j}-Q_{r}-Q_{F}-Q_{k}
$$

where $Q_{k}=1 / 2 \Gamma V^{2}$. A one-dimensional time-dependent model of the plasma layer is considered, and all parameters in Eq. (1) depend on the coordinate $y$ along the propellant face (Fig. 2). The Joule heat term is equal to $Q_{j}=j^{2} / \sigma$, where $\sigma=e^{2} n /\left(v_{\mathrm{ei}} m_{e}\right)$ and $v_{\mathrm{ei}}=\ln \Lambda Z_{i} n /\left(3 \times 10^{10} T_{e}^{3 / 2}\right)$ (Ref. 21). One can see that the plasma conductivity has only a weak dependence on the plasma density (in the Coulomb logarithm). The radiation heat flux $Q_{r}$ and particle convection heat flux $Q_{F}$ depend on the plasma density and temperature. ${ }^{8-10}$ According to Zemskov et al., ${ }^{22}$ the radiation in continuum from a $\mathrm{C}-\mathrm{F}$ plasma in the considered parameter range $\left(n=10^{22}-10^{24} \mathrm{~m}^{-3}, T_{e}=1-3 \mathrm{eV}\right)$ provides the main contribution. The radiation energy flux $Q_{r}$ includes the radiation for a continuum spectrum based on a theoretical model. ${ }^{11,23}$ The radiation term calculation is described in detail elsewhere. ${ }^{8-10}$ The particle convection flux $Q_{F}$ includes energy associated with electron and ion fluxes to the Teflon. In the steady state (floating potential), the ion and electron particle flux to the surface can be calculated as follows: $j_{i}\left(2 T_{e}+U_{\mathrm{sh}}+T_{e}\right)$. The electron temperature is assumed to be constant across the plasma layer due to a large electron thermal conductivity. ${ }^{12}$ More details about the model and the computational methods employed can be found elsewhere. ${ }^{10,13}$

The plasma layer model is combined with a heat conductivity model for the Teflon. The temperature can be calculated from the heat transfer equation,

$$
\frac{\partial T}{\partial t}=a \Delta T
$$

where $a=\lambda / C_{p} \rho$. Equation (2) is subject to the following boundary conditions at the propellant wall ${ }^{10}$ :

$$
\begin{gathered}
-\lambda \frac{\partial T}{\partial z}(z=0)=q(t)-\Delta H \cdot \Gamma-C_{p}\left(T_{s}-T_{0}\right) \Gamma \\
\lambda \frac{\partial T}{\partial z}(z=\infty)=0, \quad T(t=0)=T_{0}
\end{gathered}
$$

where $z=0$ corresponds to the dielectric surface and $q(t)$ is the density of the heat flux, consisting of the radiative and particle convection fluxes, determined according to Eq. (1). The radiation transport inside the solid Teflon was neglected in this analysis. Assuming that the thermal conductivity of the Teflon is small, we can reduce Eqs. (2) and (3) to a one-dimensional problem and, therefore, we will solve the local heat balance problem instead of the two-dimensional heat balance over the electrode surface. After the surface temperature reaches some critical temperature, material sublimation begins and ablation heat becomes significant in the energy balance. Under these conditions, the temperature profile for the ablated Teflon is exponential, ${ }^{10}$

$$
T(z)=T_{s} \exp \left(-z \Gamma C_{p} / \lambda\right)
$$

The results of the local heat balance problem inside and outside of the constricted region are shown later (see Sec. V).

\section{B. Azimuthal Nonuniformity of Discharge}

We will start with an analysis of the arc discharge nonuniformity. Typically, in previous modeling efforts, an azimuthally uniform current sheet has been assumed for simplicity. Figure 3 shows photographs recorded by a high-speed camera showing the visible 


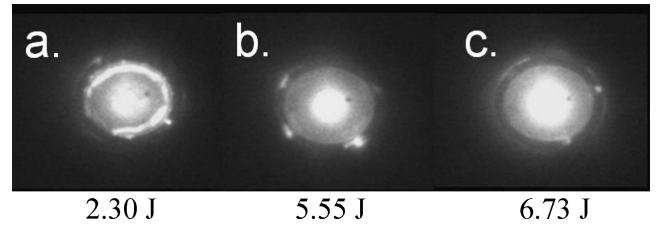

Fig. 3 Evidence of arc spoking in 6.35-mm-diam $\mu$ PPT.

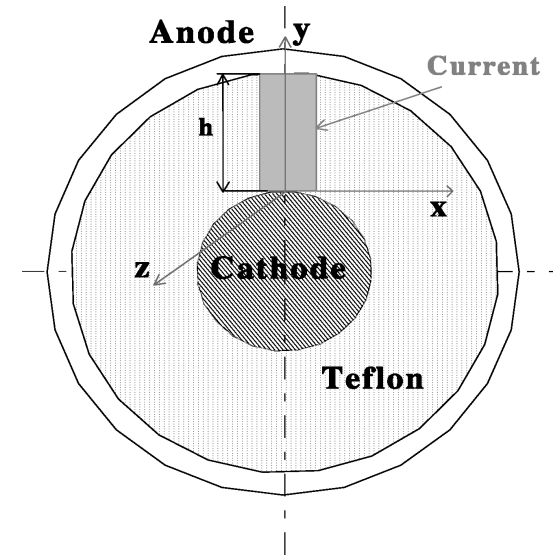

Fig. 4 Schematic of model geometry.

emission for a $\mu$ PPT firing at several energies. The camera integrated the light signal through $20 \mu \mathrm{s}$, which corresponds to the end of the current pulse for each firing. At low energies, the photographs indicate broad arc attachment around the outer electrode. As the discharge energy increases, specific locations are identified where the visible emission is localized. This is interpreted here as spoked arc attachment indicative of azimuthal nonuniformity. The spoke size varies from $20 \mathrm{deg}$ to a broad spoke of $180 \mathrm{deg}$, dependent on the conditions. Because the low-energy regime results in char formation on the $\mu \mathrm{PPT}$ surface, ${ }^{8}$ typical thruster operation is in a regime where the arc is decidedly nonuniform. If the discharge is nonuniform, the current density will increase locally. This in turn will lead to an increase in ohmic plasma heating (which is proportional to $j^{2}$ ). As a result, in the local areas of discharge concentration, the heat fluxes to the propellant surface will increase, which will in turn lead to locally high surface temperature and ablation rate. On the other hand, the ablated surface area will be smaller, and this may also affect the total ablation rate during the pulse.

\section{Current Constriction}

Several physical reasons may lead to discharge nonuniformity, such as current constriction and cathode and anode spot formation. The probability of all of these effects increases as the discharge current increases. For instance, it was shown ${ }^{14}$ that a current increase in the range of several kiloamphere leads to significant current constriction dependent on the plasma density distribution. The associated effects of the cathode and anode spot generation depend also on the current constriction. Therefore, one can expect that an increase in discharge energy (and corresponding current increase) may lead to a high probability of current constriction that will generate azimuthal nonuniformity of the discharge.

To study the possibility of current constriction under typical $\mu$ PPT conditions, we adopt the geometry shown in Fig. 4. A single current channel is considered, and therefore, all geometry effects, such as $1 / r$ dependence, are neglected. Thus, in this model the stability of a single arc spoke (Fig. 4) against constriction is investigated. We assume that the density gradients have a small effect on current constriction. The current constriction in the interelectrode region will be modeled in the framework of a steady-state hydrodynamic model,

$$
\begin{gathered}
j=(1 / \mu) \nabla B \\
j=\sigma[-\nabla \varphi-(j \times B) / e n] \\
\nabla \cdot j=0
\end{gathered}
$$

The last equation (Ohm's law) is an implicit equation and will be solved numerically by iterations to be described. It is assumed that the magnetic field has only a $z$ component and can be calculated from Ampere's law,

$$
B_{z}=-\mu \int_{0}^{x} j_{y}(x) \mathrm{d} x
$$

In component form, Eq. (6) can be written as follows:

$$
j_{x}=\sigma\left(-\frac{\partial \varphi}{\partial x}-\frac{j_{y} B_{z}}{e n}\right), \quad j_{y}=\sigma\left(-\frac{\partial \varphi}{\partial y}+\frac{j_{x} B_{z}}{e n}\right)
$$

Combining Eqs. (7) and (8) leads to the following equation for the potential distribution:

$$
\begin{gathered}
\frac{\partial^{2} \varphi}{\partial y^{2}}+\frac{\partial^{2} \varphi}{\partial x^{2}}-\frac{\beta}{h} \frac{\partial \varphi}{\partial y}-\left(\frac{\partial \varphi}{\partial x}-\beta \frac{\partial \varphi}{\partial y}\right) \frac{2 \beta\left(\beta_{0} / h\right)}{1+\beta^{2}}=0 \\
\beta=\frac{\sigma B}{e n}, \quad \beta_{0}=\frac{\sigma}{e n} \mu j_{y} h
\end{gathered}
$$

The problem reduces to solving the equation for the potential distribution in the interelectrode gap with the following boundary conditions: cathode, $\varphi=0$; anode, $\varphi=\varphi_{a}$ (discharge voltage); and centerline, $\partial \varphi / \partial x=0$.

The numerical analysis is similar to that developed previously. ${ }^{12,13}$ An iterative procedure for finding the plasma density, velocity, and potential distribution self-consistently is employed. The equation for potential [Eq. (9)] is solved numerically by iteration using the successive overrelaxation procedure. The discharge energy is used as an input parameter for this problem.

The current distribution calculation in the interelectrode gap is shown in Fig. 5 with the discharge energy as a parameter. The origin of the coordinate system corresponds to the position at the inner electrode radius (cathode, $y=0$ ). One can see that, due to the effect of the self-magnetic field, the current is constricted near the anode, leading to arc spoking as reported earlier. ${ }^{9}$ It can be seen that the increase of the discharge energy leads to stronger current constriction. For instance, the current density near the anode increases with respect to that near the cathode by a factor of five in the case of a discharge energy of about $6.7 \mathrm{~J}$ as shown in Fig. 5c.

One can expect that discharge nonuniformity in the azimuthal direction may lead to significant changes in thruster performance, such as ablation rate. Conditions for the nonuniform discharge operation will be considered later. In addition, note that current constriction affects the current continuity at the anode, that is, anode spot appearance. This effect is considered in the next section.

\section{Anode Spot Formation}

In this section, we describe a model of the near-anode region that may lead to anode spot formation. In the preceding section, it was shown that current constriction takes place under typical $\mu$ PPT conditions. Current constriction causes a significant increase of the current density near the anode, as shown in Fig. 6. Therefore, it is important to understand if current continuity can still be provided near the anode in the diffuse discharge mode. The first criterion is related to the electron thermal current near the anode, which is a function of the plasma density. ${ }^{15}$ If the thermal electron current density (which is the maximum possible electron current density) is smaller than the discharge current density near the anode, the anode current continuity cannot be provided and, therefore, anode spots 

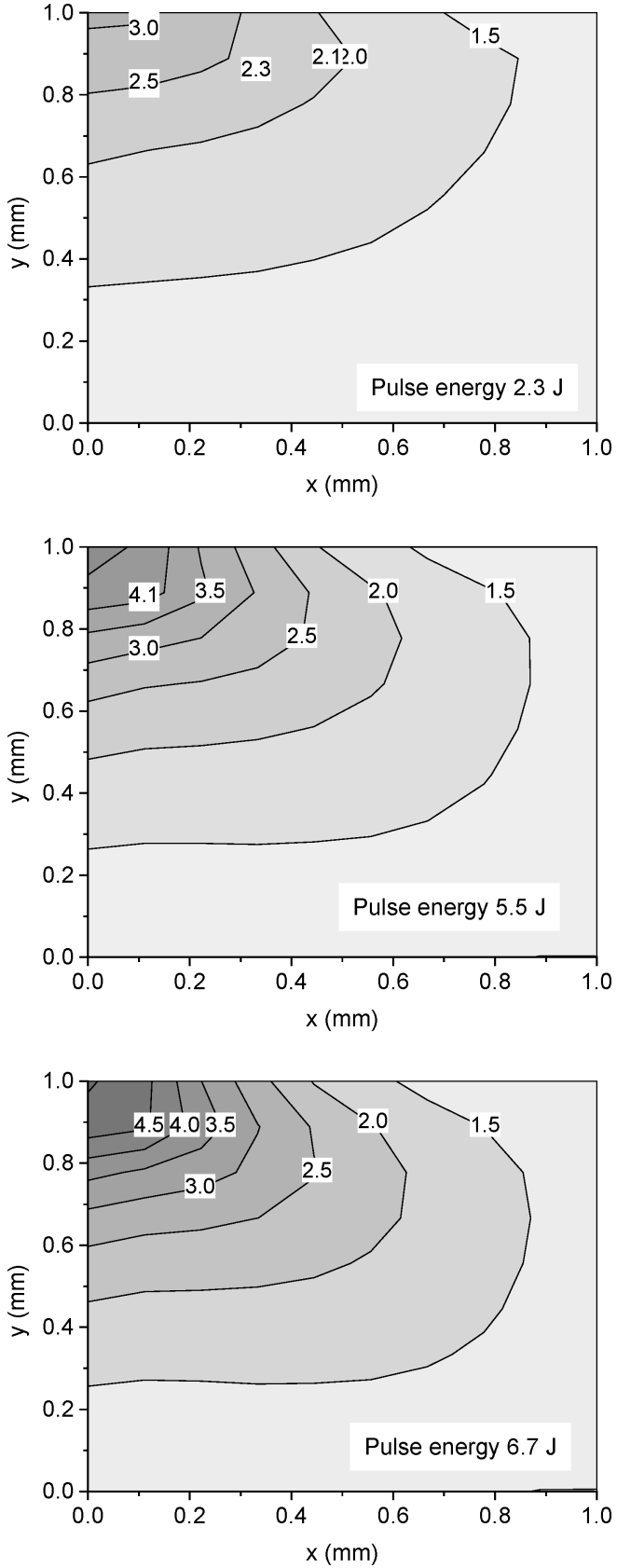

Fig. 5 Current density distribution (normalized by current density at cathode) with current constriction near upper electrode (anode, $y=1 \mathrm{~mm})$.

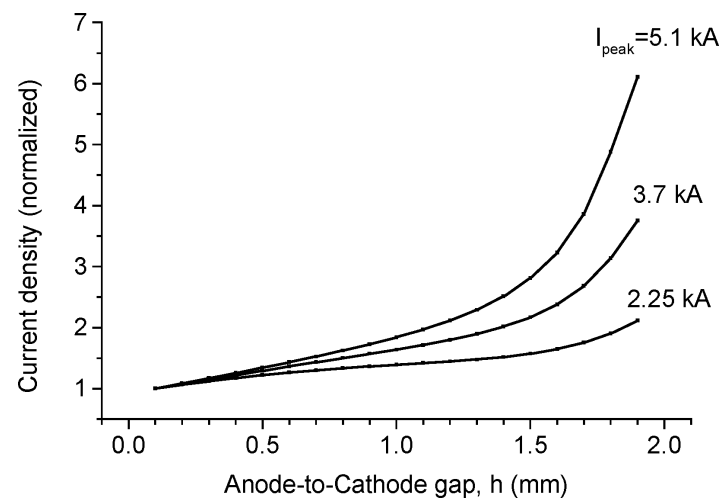

Fig. 6 Current density in constriction region (normalized by current density at cathode).

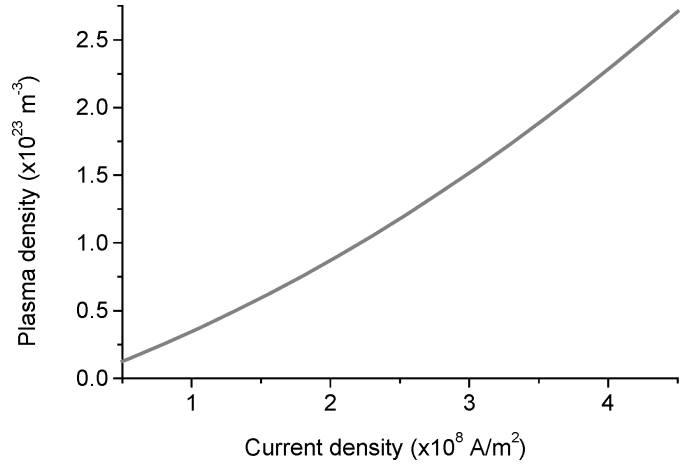

Fig. 7 Plasma density near anode as function of current density.

may appear. ${ }^{15}$ In other words, the current density increase due to current constriction cannot be supplied by the near-anode plasma in the diffuse discharge mode, thus leading to formation of the spot discharge mode. ${ }^{15}$

The calculated plasma density as a function of the current density (according to the plasma-layer model described earlier) is shown in Fig. 7. These calculations are performed for a 6.35-mm-diam $\mu$ PPT operated at $6 \mathrm{~J}$. One can see that the plasma density generally increases with current density, and therefore, plasma density can provide current continuity even in the constricted region near the anode. However, current constriction leads to an increase of the energy input to the anode and, therefore, increases the anode heating and triggers local erosion. If the density of the eroded particles (from the anode) approaches the plasma density near the anode, an anode spot may appear. ${ }^{16}$ Under this condition, the plasma density due to local evaporation from the anode becomes high so that the discharge attaches to this location. ${ }^{16}$ This leads to the following condition for the anode spot formation:

$$
n_{\mathrm{er}}=n_{e a}
$$

The thermal model of the anode used in our calculations of the anode surface temperature $T_{a}$ is similar to that for Teflon [see Eqs. (2-4)]. We use the following experimental vapor pressure curve for $\mathrm{Cu}$ :

$$
P_{\mathrm{eq}}=10^{A-B / T_{a}}
$$

where $A$ and $B$ are tabulated constants. ${ }^{17}$ Having calculated the anode surface temperature, one can calculate the equilibrium vapor pressure and the density of the eroded particles. The heat flux to the anode consists of the electron flux and can be calculated as follows ${ }^{10,19}$.

$$
q_{a}=j_{a}\left[2 T_{e}+T_{e} \ln \left(j_{a} / j_{\mathrm{eth}}\right)\right]
$$

It is assumed [in Eq. (11)] that the electrons have a Maxwellian energy distribution and the anode sheath potential drop is determined by the ratio of the anode current density to the thermal electron current density. ${ }^{15}$

The dependence of the ratio of the eroded atom number density (from the anode) to the plasma density near the anode is shown in Fig. 8 as a function of the combination of the peak current and interelectrode distance with peak current as a parameter. When this ratio reaches 1, one can expect that anode spots will appear [Eq. (10)]. This dependence demonstrates how the criterion for azimuthal uniformity is connected with anode current constriction and anode spot appearance. Conditions resulting in anode spot generation can exist dependent on the peak discharge current and the interelectrode distance. Clearly smaller discharge current and interelectrode distance help to avoid anode spots (and, therefore, azimuthal nonuniformity). Based on these calculations, the critical peak discharge current for a given thruster geometry or critical thruster size for given peak discharge current can be estimated leading to thruster geometry and discharge optimization, that is, creation of conditions for an azimuthally uniform discharge. This model prediction agrees 
Table 1 Comparison of the ablated mass per pulse for various PPTs

\begin{tabular}{lcccc}
\hline \hline Thruster & $\begin{array}{c}\text { Ablation } \\
\text { rate, } \mu \mathrm{g}\end{array}$ & Pulse energy, J & $\begin{array}{c}\text { Specific ablation } \\
\text { rate, } \mu \mathrm{g} / \mathrm{J}\end{array}$ & Reference \\
\hline $\begin{array}{l}\text { LES 8/9 } \\
\mu \text { PPT, }\end{array}$ & 26 & 20 & 1.3 & 24 \\
$\quad$ experiment & $6-10$ & 2.5 & $2.4-4$ & 5,6 \\
$\begin{array}{l}\mu \text { PPT, } \\
\quad \text { simulation }\end{array}$ & $4-14$ & 2.5 & $1.6-5.6$ & This work \\
$\begin{array}{l}\text { Elecrothermal } \\
\text { PPT (PPT-4) }\end{array}$ & $30-40$ & 10 & $3-4$ & 25 \\
\hline \hline
\end{tabular}

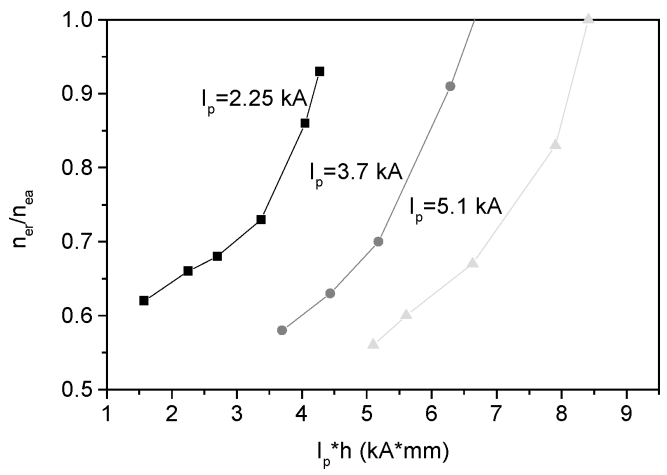

Fig. 8 Anode spot criterion.

qualitatively with experimentally observed discharge constriction near the anode as shown in Fig. 3 and described in more detail elsewhere. ${ }^{9}$

Note that in general other factors such as propellant surface nonuniformities and roughness of the electrode surface may lead to discharge nonuniformity. However, our observations suggest that azimuthal nonuniformities in a $\mu$ PPT have a regular structure as shown in Fig. 3 and, therefore, are not likely related to the described effects that have a stochastic nature.

\section{Ablated Mass in Nonuniform Discharge Mode}

If the discharge is nonuniform, the current density will increase locally. This in turn will lead to an increase of the heating of the plasma due to ohmic heating (which is proportional to $j^{2}$ ). As a result, in the local areas of the discharge concentration, the heat fluxes to the propellant surface will increase. This will in turn lead to locally high surface temperature and ablation rate. On the other hand, the ablated surface area will be smaller, and this may also affect the total ablation rate during the pulse. Let us now test this qualitative description using the already described model (Sec. II). The simplest approach is to use a current density enhancement factor due to discharge nonuniformity as an input condition.

The calculated ablation rate in this case is shown in Fig. 9. As expected, the ablation rate increases with current density enhancement. However, some saturation of the ablation rate with current density increase is predicted. This saturation is because the propellant surface exposed to the ablation decreases accordingly, and although the ablation rate in the area of the discharge concentration is higher, the total ablation rate tends to saturate.

The possibility of azimuthal nonuniformity of the discharge in the $\mu$ PPT leads to very high ablation rate as compared to largescale PPTs, such as the LES 8/9. Comparison of the ablation rates for different thrusters is shown in Table 1 (see Refs. 5, 6, and 24). For reference, the ablation rate for the electrothermal PPT (PPT-4, Ref. 25) is also shown.

It can be seen that the ablation rate in the $\mu \mathrm{PPT}$ is much higher than that in the LES 8/9. This effect can be explained in terms of nonuniform ablation, that is, azumuthal nonuniformity and associated with this current density increase, as discussed in the preceding section.

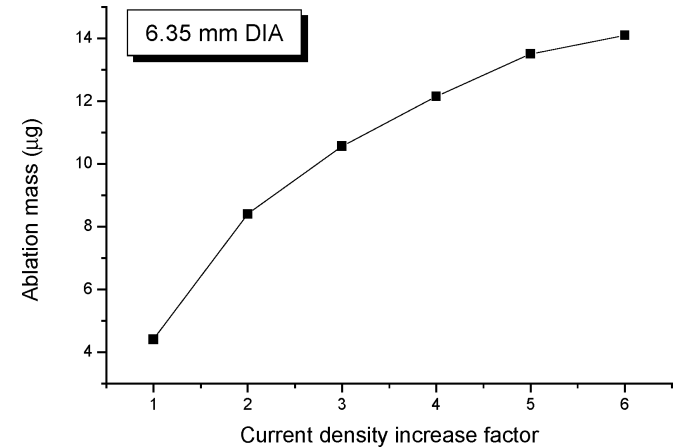

Fig. 9 Ablation mass (per single pulse) dependence on current density increase due to discharge nonuniformity in azimuthal direction.

\section{Teflon Temperature Distribution in a Nonuniform Discharge}

In this section, we describe the calculation of the Teflon surface temperature in the case when the current constriction phenomenon is strong. We calculate the Teflon surface temperature taking into account current density growth in the constricted areas (spots). The Teflon surface temperature is calculated [Eqs. (24)] inside the discharge spot and outside of the spot. The calculations inside the spot correspond to a discharge peak current of $6 \mathrm{kA}$ (4.5-J pulse energy) that leads to current constriction in the 6.35-mm-diam $\mu$ PPT (Figs. 4 and 5). The $\mu$ PPT geometry and operational conditions are described in detail elsewhere. ${ }^{26}$ We will assume that there is current conductivity in the regions outside of the constriction area and the total current fraction outside of the constricted region is used as a parameter (varied between 0.1 and 0.5 of the total current), that is, $I_{\text {out }}=(0.1-0.5) I$. During the discharge pulse, the Teflon surface temperature distribution is calculated by solving the heat transfer problem [Eqs. (2-4)]. After the pulse (about $25 \mu \mathrm{s}$ ), heat propagates inside the propellant and the surface temperature cooling can be described according to the following expression $^{18,19}$ :

$$
T_{s}=T_{0}+\left(t_{p} / t\right)^{0.5}\left(T_{\mathrm{sp}}-T_{0}\right)
$$

This temperature distribution is obtained after assuming that there are no heat sources after the discharge pulse; thus, Eq. (12) is valid after $t>25 \mu \mathrm{s}$ that is, $t_{p}=25 \mu \mathrm{s}$.

Teflon thermal conductivity along the propellant surface is not taken into account because it is relatively small and does not affect the results according to our estimates. This allows us to reduce the analysis to the local solution of the heat transfer problem in the constricted region and outside of it.

The calculated results are shown in Fig. 10. One can see that current increase due to the constriction effect significantly affects the Teflon surface temperature. It can be seen that in the constricted region (anode spot) the peak Teflon surface temperature reaches about $1070 \mathrm{~K}$, whereas outside of the constricted region it is about $900 \mathrm{~K}$. Note that this difference can be considered very significant due to strong effect of the ablation [see Eq. (4)] in the temperature balance that limits the temperature increase. It is shown subsequently that a similar difference between two such cases was obtained experimentally.

For comparison, the experimental results taken from Ref. 26 are shown in Fig. 10. The experiment was conducted for a 4.5-J discharge pulse with a peak current of about $6 \mathrm{kA}$. In Fig. 10, the error bars are shown for after the discharge is finished and indicate the measurement uncertainty at a time when plasma radiation is not contributing. Surface temperature is measured by photovoltaic infrared detectors ${ }^{20}$ with an active area of $80 \times 80 \mu \mathrm{m}$ square. The diameter of the $\mu$ PPT is $6.35 \mathrm{~mm}$, and so the spatial resolution of the detectors is small on the propellant surface. The detector is imaged $1: 1$ on a point halfway between the inner and outer electrode radii. Movement of arc constrictions around the azimuth of the propellant face can affect the measured temperatures, and a statistical spread of 


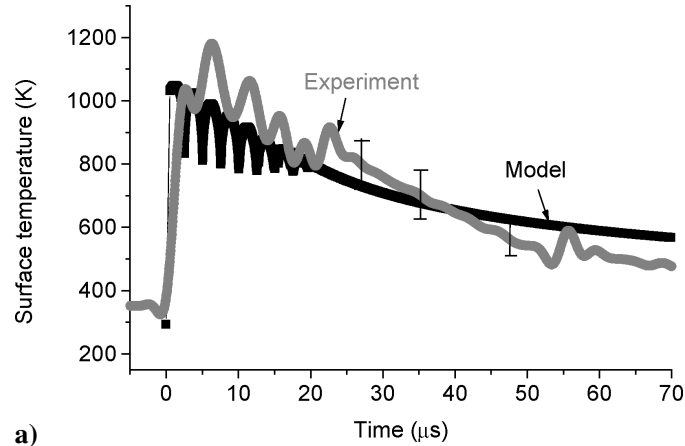

a)

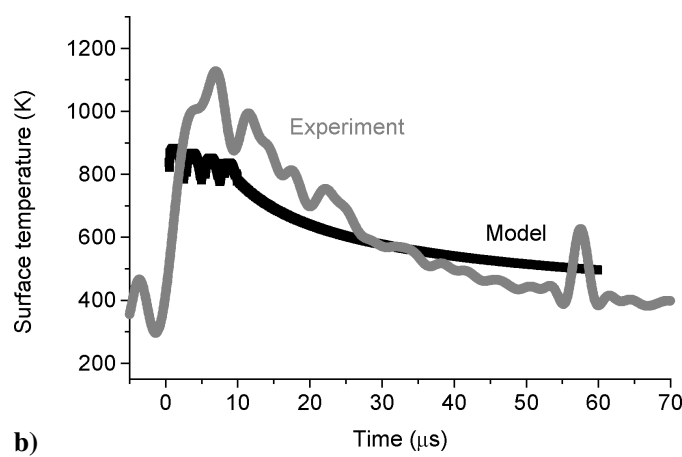

b)

Fig. 10 Teflon surface temperature a) inside spot and b) outside of spot. Comparison between simulations and experiment.

temperatures is expected because the measurement location is stationary over many pulses. Experimental results are reliable only after the discharge pulse is gone, which is approximately $25 \mu$ s after discharge initiation. During the discharge, the experimental signal is affected by the plasma radiation. Experimental measurements shown in Fig. 10 are assumed to be in two distinct locations: near the arc constriction and far from the arc constriction (Fig. 3). However, there was no synchronized camera available during these experiments to confirm this assumption fully. We conjecture that near the arc constriction the surface temperature will be higher due to increased particle flux to the surface. Far from the arc, it is assumed the surface temperature will be lower because heat transfer is dominated by radiation from the arc. Absorption, especially in the infrared, is low for Teflon. Predictions shown in Fig. 10 demonstrate these hypotheses and match well with the highest (Fig. 10a) and lowest (Fig. 10b) experimental temperatures measured in the data set. This evidence suggests that the conjecture is true and variations in surface temperature measurement within the ranges measured can be explained by different heating mechanisms and arc proximity to the measurement location. Generally good agreement between the experimental results and simulation is obtained for the entire pulse duration for both cases.

\section{Propellant Charring Dependence on the Discharge Energy}

It was found previously that a low energy-to-area ratio leads to Teflon surface charring. ${ }^{27,28}$ Previous analysis suggests that the charring is associated with carbon atom and ion backflux. ${ }^{28}$ Carbon deposition on the Teflon surface leads to film growth. Note that the carbon film is more difficult to evaporate in comparison to the Teflon, and therefore, when the carbon layer is developed it cannot be evaporated under the typical PPT conditions. Initial discontinuous carbon film growth can be understood by study of discontinuous film growth. ${ }^{29}$ Carbon film growth (initial island growth) depends on the carbon flux to the surface. A typical image of the carbon-based film grown on the Teflon surface is shown in Fig. 11.

Carbon backflux depends on the conditions at the Knudsen-layer edge as was considered in Ref. 30. In this paper, we present a calculation for a specific geometry (3.6-mm outer diameter $\mu$ PPT). The

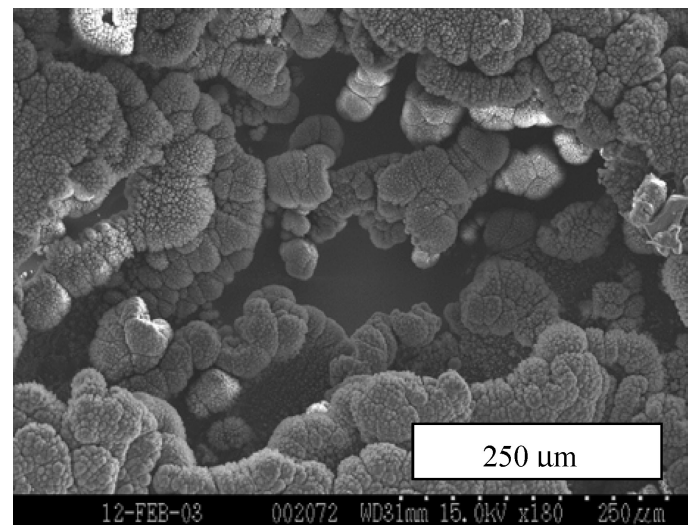

Fig. 11 Scanning electron microscope image of charred area at Teflon surface ( $\times 180$ magnification), 6.35-mm-diam $\mu$ PPT, 1-J pulse energy.

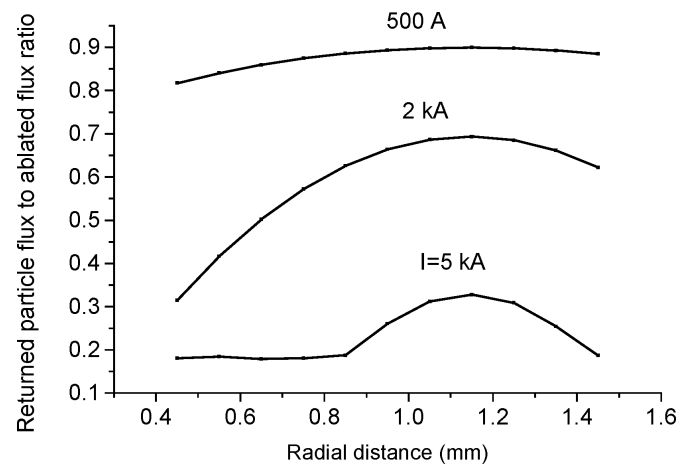

Fig. 12 Dependence of backflux on radial position with discharge current as parameter.

calculated backflux depends on the current density in the vicinity of the Teflon surface. Higher current density leads to plasma acceleration, and as a result the velocity at the Knudsen-layer edge increases up to the limit of the sound speed. ${ }^{31}$ This means that the particle backflux decreases when current density increases. Because of the current spread in the near-field plume, the current density is smaller in the region between the electrodes in comparison to the current density near the electrodes. ${ }^{28}$ Therefore, one can expect the smallest velocity and as a result the largest backflux in this area. These results are shown in Fig. 12. One can see that the highest backflux fraction is predicted to be in the middle of the propellant surface between the electrodes, and therefore, one can expect that the highest carbon film growth rate will be in this region. This corresponds to experimental findings. ${ }^{20,27}$ In addition, it can be seen that to achieve a low backflux fraction and, therefore, a low possibility of carbon char formation a high discharge current, that is, high pulse energy, is required.

\section{Effect of Discharge Nonuniformity on Thrust Bit and Specific Impulse}

In this section, we consider the effect of discharge nonuniformity on $\mu$ PPT performance characteristics such as impulse bit and specific impulse. Previously it was shown that the $\mu$ PPT exhibits a primarily electromagnetic acceleration mechanism. ${ }^{13}$ In the electromagnetic approximation, it is assumed that the entire work is done by the circuit to accelerate the moving plasma cloud. ${ }^{32,33}$ In this analysis, we neglect losses such as eddy current losses. In this case, the total impulse that can be generated during the pulse can be estimated as follows:

$$
\frac{\mathrm{d}(\Delta M V)}{\mathrm{d} t}=\frac{b}{2} I^{2}
$$


where $b$ is the theoretical effective specific inductance. Note that this equation provides an expression for the maximum force regardless of the details of the plasma interaction with the electromagnetic force. ${ }^{32}$ If the exit velocity is $V_{e}$, we can estimate the specific impulse from the impulse bit according to the relation

$$
I_{\mathrm{bit}}=\Delta M V_{e}=\Delta M I_{\mathrm{sp}} g
$$

It was shown that the total ablated mass increases with current constriction. Impulse bit $I_{\text {bit }}$ does not depend on the constriction effect because it depends on the total current. Therefore, specific impulse will decrease as a result of constriction, showing that discharge nonuniformity leads to degradation of the thruster performance characteristics and, therefore, should be avoided.

\section{Summary}

In this paper we considered some peculiarities related to $\mu \mathrm{PPT}$ operation, in particular, dependence on the energy pulse and thruster geometry. It was shown that both discharge energy (peak current) and thruster size affect significantly the discharge uniformity (azimuthal or radial). Azimuthal nonuniformity relates to the current constriction and anode spot formation phenomena. This happens when the discharge current or thruster size exceed some critical value. Discharge nonuniformity leads to a much higher ablation rate and causes degradation of the specific impulse. On the other hand, small discharge current leads to strong Teflon surface carbonization (charring) and radial nonuniformity, which in turn leads to thruster failure. The primary mechanism of the charring formation was identified to be related to carbon backflux.

Thus, the thruster size and discharge energy can be optimized by trading between two conflicting requirements of a large pulse energy (to prevent charring) and a small discharge energy (to prevent current constriction). Because the charring phenomenon is completely intolerable and leads to thruster failure, the optimal discharge energy should be chosen somewhere near the spot formation limit. An example of the calculated limit for discharge energy selection is shown in Fig. 8. These results suggest the following design path. For a given thruster size (which could be fixed from various system considerations), one can calculate the optimal discharge energy by considering the current constriction phenomenon using the simple approach that was developed in this paper. This will lead to the selection of the optimal energy necessary for preventing spot mode discharge appearance. On the other hand, propellant charring effects should be studied for the selected discharge energy, making sure that the charring phenomenon is completely eliminated.

\section{Acknowledgments}

The first two authors gratefully acknowledge financial support by the Air Force Office of Scientific Research through Grant F4962002-1-0084 and by the Air Force Research Laboratory (AFRL). We also acknowledge D. Bromaghim (AFRL) and V. Hruby and L. Byrne (Busek) for valuable discussions. The authors also wish to acknowledge support from ERC, Inc. The experimental work was performed at the Electric Propulsion Laboratory at Edwards Air Force Base, California.

\section{References}

${ }^{1}$ Spores, R. A., Cohen, R. B., and Birkan, M., "The USAF Electric Propulsion Program," Proceeding of the 25th International Electric Propulsion Conference, Vol. 1, Electric Rocket Propulsion Society, Worthington, OH, 1997, p. 1

${ }^{2}$ Micropropulsion for Small Spacecraft, edited by M.M. Micci and A.D. Ketsdever, AIAA, Reston VA, 2000.

${ }^{3}$ Dunning, J. W., Benson, S., and Oleson, S., "NASA's Electric Propulsion Program," 27th International Electric Propulsion Conference, Electric Rocket Propulsion Society, Worthington, OH, 2001; also Paper IEPC-01002 .

${ }^{4}$ Zakrzwsky, C., Benson, S., Sanneman, P., and Hoskins, A., "On-Orbit Testing of the EO-1 Pulsed Plasma Thruster," AIAA Paper 2002-3973, July 2002.
${ }^{5}$ Spanjers, G. G., White, D., Schilling, J., Bushman, S., Lake, J., and Dulligan, M., "AFRL MicroPPT Development for the TechSat21 Flight," 27th International Electric Propulsion Conference, Electric Rocket Propulsion Society, Worthington, OH, 2001; also Paper IEPC-01-166.

${ }^{6}$ Spanjers, G. G., Bromaghim, D. R., Lake, J., Dulligan, M., White, D., Schilling, J. H., Bushman, S. S., Antonsen, E. L., Burton, R. L., Keidar, M., and Boyd, I. D., "AFRL MicroPPT Development for Small Spacecraft Propulsion," AIAA Paper 2002-3974, July 2002.

${ }^{7}$ Simon, D. H., and Land, H. B., "Pulsed Plasma Thrusters for Micropropulsion," AIAA Paper 2003-5170, July 2003.

${ }^{8}$ Keidar, M., Boyd, I. D., Gulczinski, F. S., Antonsen, E. L., and Spanjers, G. G., "Analyses of Teflon Surface Charring and Near Field Plume of a Micro-Pulsed Plasma Thruster," 27th International Electric Propulsion Conference, Electric Rocket Propulsion Society, Worthington, OH, 2001; also Paper IEPC-01-155.

${ }^{9}$ Keidar, M., Boyd, I. D., Antonsen, E. L., and Spanjers, G. G., "Progress in Development of Modeling Capabilities for a Micro-Pulsed Plasma Thruster," AIAA Paper 2003-5166, July 2003.

${ }^{10}$ Keidar, M., Boyd, I. D., and Beilis, I. I., "Model of an Elecrothermal Pulsed Plasma Thruster," Journal Propulsion and Power, Vol. 19, No. 3, 2003, pp. 424-430.

${ }^{11}$ Raizer, Y. P., Gas Discharge Physics, Nauka, Moscow, 1987 (in Russian).

${ }^{12}$ Keidar, M., Boyd, I. D., and Beilis, I. I., "Ionization and Ablation Phenomena in an Ablative Plasma Accelerator," Journal of Applied Physics, Vol. 96, No. 10, 2004, pp. 5420-5428.

${ }^{13}$ Keidar, M., Boyd, I. D., Antonsen, E. L., and Spanjers, G. G., "Electromagnetic Effects in the Near Field Plume Exhaust of a Micro-Pulsed Plasma Thruster," Journal of Propulsion and Power, Vol. 20, No. 6, 2004, pp. 961-969.

${ }^{14}$ Beilis, I., Keidar, M., Boxman, R. L., and Goldsmith, S., "Theoretical Study of Plasma Jet Expansion in a Magnetic Field in a Disc Anode Vacuum Arc," Journal of Applied Physics, Vol. 83, No. 2, 1997, pp. 709-717.

${ }^{15}$ Keidar, M., Schulman, M. B., and Taylor, E., "Model of a Diffuse Column Vacuum Arc With Cathode Jets Burning in Parallel with a High-Current Plasma Column," IEEE Transaction on Plasma Science, Vol. 32, No. 2, 2004, pp. 783-791.

${ }^{16}$ Wickert, C., and Egli, W., "Theoretical Analysis of the Current and Energy Flow to the Anode in the Diffuse Vacuum Arc," IEEE Transaction on Plasma Science, Vol. 17, No. 5, Oct. 1989, pp. 649-652.

${ }^{17}$ Duschman, S., Scientific Foundations of Vacuum Technique, Wiley, New York, 1962.

${ }^{18}$ Turchi, P. J., "Directions for Improving PPT Performance," Proceeding of the 25th International Electric Propulsion Conference, Vol. 1, Electric Rocket Propulsion Society, Worthington, OH, 1998, pp. $251-258$.

${ }^{19}$ Keidar, M., Boyd, I. D., and Beilis, I. I., "Electrical Discharge in the Teflon Cavity of a Co-axial Pulsed Plasma Thruster," IEEE Transaction on Plasma Science, Vol. 28, No. 2, 2000, pp. 376-385.

${ }^{20}$ Antonsen, E. L., Burton, R. L., Spanjers, G. G., and Spores, R. A., "Time-Resolved Surface Temperature Measurement for Pulsed Ablative Thrusters," Proceeding of the 28th International Electric Propulsion Conference, Electric Rocket Propulsion Society, Worthington, OH, 2003; also Paper IEPC-03-282.

${ }^{21}$ Braginsky, S. I., Review of Plasma Physics, edited by M.A. Leontovich, Vol. 1, Consultant Bureau, New York, 1965.

${ }^{22}$ Zemskov, A. I., Prut, V. V., and Khrabrov, V. A., "Pulsed Discharge in Dielectric Chamber," Soviet Physics-Technical Physics, Vol. 17, 1972, pp. 285-289.

${ }^{23}$ Kozlov, G. I., Kuznetsov, V. A., and Masyukov, V. A., "Radiative Losses by Argon Plasma and the Emissive Model of a Continuos Optical Discharge," Soviet Physics-JETP, Vol. 39, No. 3, 1974, pp. 463-468.

${ }^{24}$ Vondra, R. J., Thomassen, K., and Solbes, A., "Analysis of Solid Teflon Pulsed Plasma Thruster," Journal Spacecraft and Rockets, Vol. 7, Dec. 1970, pp. 1402-1406.

${ }^{25}$ Bushman, S. S., and Burton, R. L., "Heating and Plasma Properties in a Coaxial Gasdynamic Pulsed Plasma Thruster," Journal of Propulsion and Power, Vol. 17, No. 5, 2001, pp. 959-966.

${ }^{26}$ Antonsen, E. L., Ph.D. Dissertation, Dept. of Aerospace Engineering, Univ. of Illinois, Urbana-Champaign, IL, May 2004

${ }^{27}$ Gulczinski, F., Dulligan, M., Lake, J., and Spanjers, G. G., "Micropropulsion Research at AFRL," AIAA Paper 2000-3255, July 2000. 
${ }^{28}$ Keidar, M., Boyd, I. D., Antonsen, E., Gulchinski, F., and Spanjers, G. G., "Propellant Charring in Pulsed Plasma Thrusters," Journal Propulsion and Power, Vol. 20, No. 6, 2004, pp. 978-984.

${ }^{29}$ Levchenko, I., Korobov, M., Romanov, M., and Keidar, M., "Ion Current Distribution on a Substrate During Nano Structures Formation," Journal Physics D: Applied Physics, Vol. 37, May 2004, pp. 1690-1695.

${ }^{30}$ Keidar, M., Fan, J., Boyd, I. D., and Beilis, I. I., "Vaporization of Heated Materials into Discharge Plasmas," Journal of Applied Physics, Vol. 89, No. 6, 2001, pp. 3095-3098.
${ }^{31}$ Keidar, M., and Boyd, I. D., "Ionization Non-equilibrium and Ablation Phenomena in a Micro-Pulsed Plasma Thruster," AIAA Paper 2002-4275, July 2002.

${ }^{32}$ Artsimovich, A. A., Luk'ynov, S. Y., Podgornyi, I. P., and Chuvatin, S. A., "Electrodynamic Acceleration of the Plasmoid," Soviet Physics—JETP, Vol. 33, No. 1, 1957, p. 3.

${ }^{33}$ Keefer, D., and Rhodes, R., "Electromagnetic Acceleration in Pulsed Plasma Thrusters," Proceeding of the 25th International Electric Propulsion Conference, Vol. 1, Electric Rocket Propulsion Society, Worthington, OH, 1998, pp. 251-258. 